\title{
Conversion of $n-3$ polyunsaturated fatty acids (PUFAs) and incorporation of docosahexaenoic acid (DHA) in cultured neural cells
}

\author{
Jean-Marc ALESSANDRI \\ Bénédicte LANGELIER \\ Marie-Hélène PERRUCHOT \\ Audrey EXTIER \\ Fabien PIFFERI \\ Mélanie JOUIN \\ Serge DELPAL \\ Monique LAVIALLE \\ Philippe GUESNET
}

Unité de Nutrition et Régulation Lipidique des Fonctions Cérébrales (Nu.Ré.Li.Ce), Institut National de la Recherche Agronomique (INRA), CRJ, 78352 Jouy-en-Josas cedex <jean-marc.alessandri@jouy.inra.fr>

\begin{abstract}
Docosahexaenoic acid (DHA, 22:6n-3) in membrane phospholipids originates from dietary intake of preformed DHA and from conversion of its essential precursor $\alpha$-linolenic acid (ALA, 18:3n-3). Cultured cells, especially nervous cells, are increasingly used to explore the uptake, metabolism and gene transcription effects of $n-3$ fatty acids, raising the question of the specific metabolic fate of different fatty acids and of the physiological relevance of their concentrations in the culture medium. This paper reports experimental data that 1) compare the dose-dependent incorporation of preformed DHA into the ethanolamine phosphoglycerolipids (EPG) of neural and cerebral endothelial cells in culture with that of the developing rat brain, 2) evaluate the pathway of DHA synthesis from ALA, eicosapentaenoic acid (EPA, 20:5n-3) or $n-3$ docosapentaenoic acid (DPA, 22:5n-3) in a model of neuronal cells, the SH-5YSY human neuroblastoma cells, and 3) characterize in these cells the mRNA expression profile of genes involved in the fatty acid metabolism. The incorporation of preformed DHA in EPG followed, both in vivo and in vitro, a dose-response curve from which two parameters were drawn: the DHAmax, i.e. the plateau-value of the linearized dose-response curve (expressed in weight $\%$ of total fatty acids), and the $D H A 50$, the concentration of DHA in the diet or in the culture medium corresponding to an incorporation of DHA in EPG equal to one-half the DHAmax. The ratio of DHAmax to DHA5O reflects the propensity (so-called the 'avidity' for DHA) of cells or tissues to incorporate the exogenous DHA. The DHAmax and the DHAmax/DHA5O ratio values of SH-SY5Y cells and of rat brain endothelial cells in culture were compared to those of the frontal cortex and hippocampus of rats chronically deficient in $n-3$ fatty acids and supplemented with preformed DHA. The same DHAmax/DHA5O ratio values were found in SH-SY5Y (5.2) cells and in rat brain areas (5.1-5.7) when the DHA doses were expressed in $\mu \mathrm{mol}$ DHA/liter of culture medium and in $\mu \mathrm{mol} D H A / 10 \mathrm{~g}$ diet, respectively. The SH-SY5Y cells were able to produce neoformed EPA, DPA and DHA from supplemental ALA. The incorporation of neoformed EPA and DPA in $E P G$ followed a dose-response saturating curve, while that of DHA was bell-shaped. The different pattern of neoformed DPA and neoformed DHA suggests that the conversion pathway was limited at the terminal step of DHA synthesis. The mRNA profile showed that two enzymes of the peroxisomal $\beta$-oxidation system, the L-and D-bifunctional proteins, were expressed at lower levels than those of the endoplasmic reticulum pathway (46-desaturase). These data show that incorporation of preformed DHA in cultured neuroblastoma cells match physiological values, indicating that DHA uptake, acyl-CoA activation, and phospholipid acyltransferases are active. However, the synthesis and incorporation of newly formed DHA in SH-SY5Y cells responds to a critical concentration-window of precursors which could originate from the low basal expression level of peroxisomal enzymes.
\end{abstract}

Key words: docosahexaenoic acid (DHA), endothelial cells, ethanolamine phosphoglycerolipids (EPG), n-3 PUFA conversion, peroxisomal enzymes, rat brain, SH-SYSY neuroblastoma cells

$[1,2]$. In human infants, the $n-3$ supplies of maternal milk or of milk replacers also have an impact on the DHA contents in nervous tissues and may produce transient (but significant) outcomes on the mental development and maturation of visual function [3]. DHA is directly provided by dietary animal fats or is synthesized from its metabolic precursors, $\alpha$-linolenic acid (ALA, 18:3n-3), eicosapentaenoic acid (EPA, 20:5n-3) or docosapentaenoic acid (DPA, 22:5n-3). The conversion of
ALA, the essential dietary precursor, occurs in the endoplasmic reticulum, producing the downstream long-chain metabolites EPA, DPA and tetracosahexaenoic acid (THA, 24:6n-3) (figure 1). THA is not incorporated into membranes but is transferred to the peroxisomes to be shortened in DHA through one cycle of $\beta$-oxidation (figure 1). Increasing the ALA intakes has been considered as a possible way of increasing the DHA status in human. However, clinical studies in adults showed that ALA 


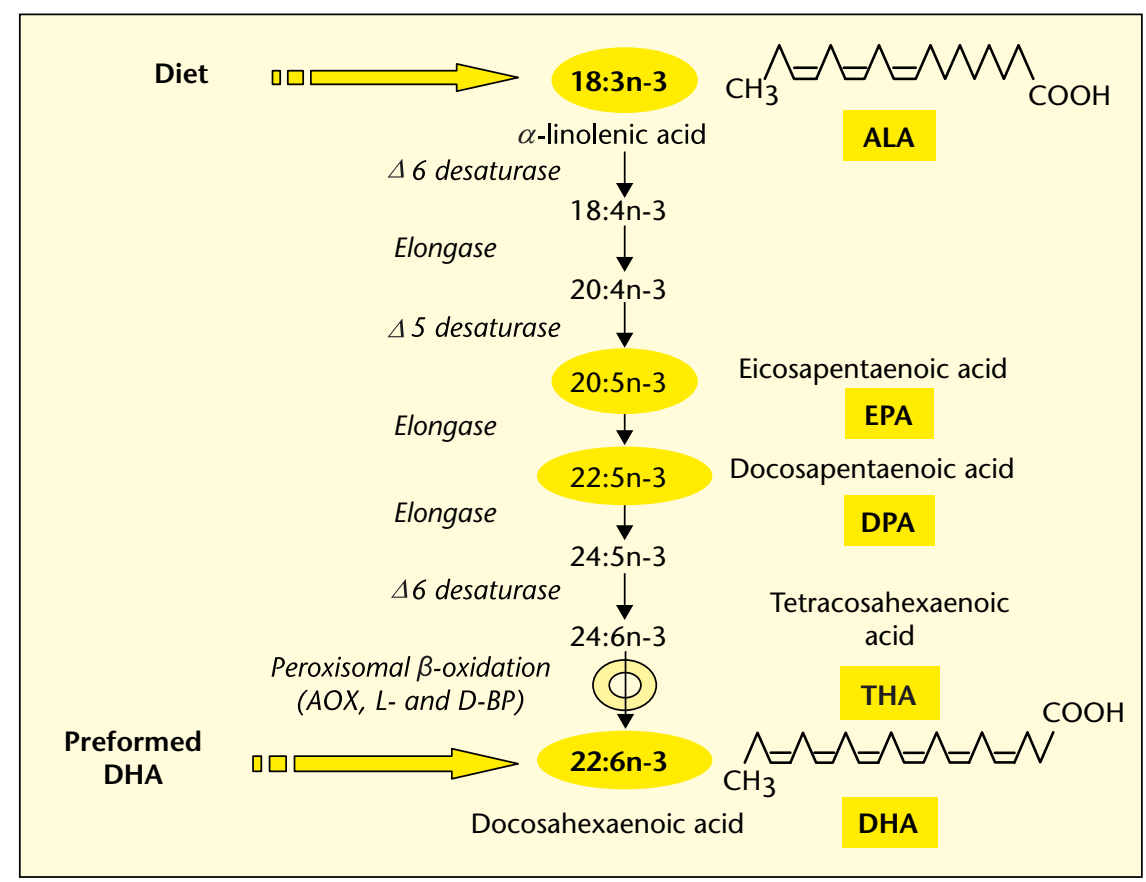

Figure 1. Metabolic pathway of bioconversion of $n-3$ polyunsaturated fatty acids (PUFAs).

The conversion of ALA (18:3n-3) occurs in the Endoplasmic Reticulum to successively produce eicosapentaenoic acid (EPA, 20:5n-3), docosapentaenoic acid (DPA, 22:5n-3) and tetracosahexaenoic acid (THA, 24:6n-3). THA is then transferred to the peroxisomes to be $\beta$-oxidized in DHA involving several specific enzymes (Acyl-CoA Oxidase (AOX) and $L$ - and $D$-bifunctional proteins ( $L$ - and $D-L B P)$ ).

supplementation with as much as $15 \mathrm{~g}$ per day did not increase DHA in plasma phospholipids, but increased EPA and DPA (reviewed in [4])

(figure 2). Moreover, EPA concentration in plasma (not DHA) increased in response to dietary EPA. These metabolic responses have

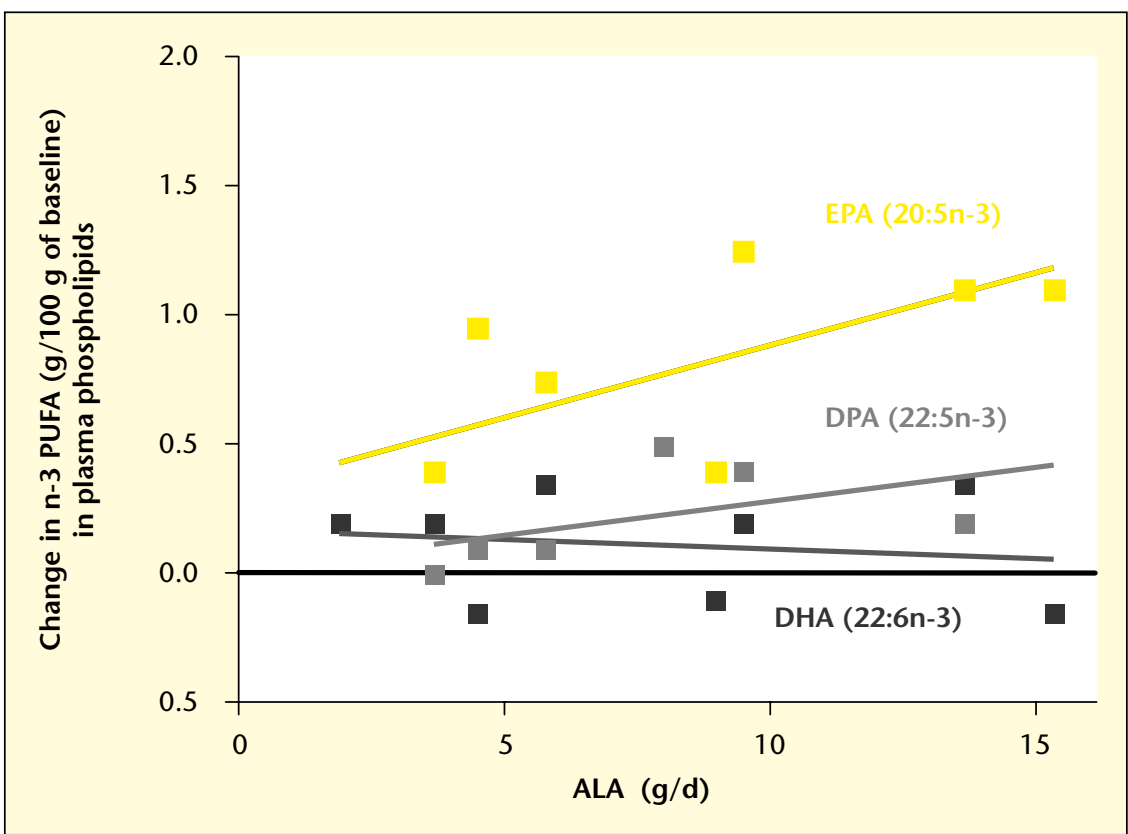

Figure 2. $\alpha$-linolenic acid (ALA, 18:3n-3) intake and n-3 PUFA plasma status in human adults. Analysis by regression of the effect of increasing dietary intake of supplemental ALA on the variation of eicosapentaenoic acid (EPA, 20:5n-3), docosapentaenoic acid (DPA, 22:5n-3) and docosahexaenoic acid (DHA, 22:6n-3) concentrations in plasma phospholipids (adapted from [4]). Each symbol corresponds to a clinical study. been related to the low capacity of conversion of ALA to DHA. Studies using stable isotope tracers in healthy adults have estimated that the conversion of ALA to EPA is around $5 \%$ of the ALA intake whereas that of ALA to DHA accounts for much less than $0.5 \%$ [5]. The reasons for this low conversion rate are not clearly elucidated. The first hypothesis is that ALA itself may mitigate DHA synthesis by competing with tetracosapentaenoic acid (24:5n-3) for $\Delta 6$ desaturation and preferential production of 18:4n-3 over that of THA [6]. Besides, active channeling of ALA towards mitochondrial $\beta$-oxidation would reduce its entry into the pathway of long-chain synthesis [5]. However, the two hypothesis seem contradictory since they mean that the entry of ALA either into the conversion pathway or into the oxidative pathway both will result in reduction of DHA synthesis. The third hypothesis is that the peroxisomal pathway could form a limiting step: the peroxisomal $\beta$-oxidation of THA requires complex movements of the $22 \mathrm{C}$ - and 24C-PUFAs on both sides of the endoplasmic reticulum and peroxisomal compartments, and it needs specific peroxisomal enzymes which could be low in activity [7].

To address these questions, we studied the $n-3$ PUFA metabolism in a neural cell model, the SH-SY5Y neuroblastoma cells which are derived from the neural crest of the human embryo and exhibit neuronal morphological features (figure 3 ). In a first approach, we compared the dosedependent incorporation of preformed DHA into the phospholipids of SH-SY5Y cells, of rat brain areas (frontal cortex and hippocampus) and of rat brain endothelial cells, and we evaluated the capacity of SH-SY5Y cells to produce neoformed DHA from its metabolic precursors, ALA, EPA and DPA. We studied the potential of SH-SY5Y cells to express genes involved in the fatty acid metabolism by quantifying the mRNAs of key enzymes located at the endoplasmic reticulum level, the FA-CoA ligase (FACL3), the $\Delta 6$ desaturase ( $\triangle 6-D)$ and the elongase of very long chain fatty acids (ELOVL4), and at the peroxisomal level, the acyl-CoA oxidase (AOX) and the two bifunctional proteins, LBP and DBP. The mRNAs of cognate transcription factors which regulate the transcription of lipid metabolism genes, the peroxisome proliferator-activated receptors (PPARs) and the retinoid $X$ receptor (RXR), and the mRNAs of enzymes not directly involved in the DHA synthesis but in the synthesis of etherphospholipids or of phospholipid classes (peroxisomal dihidroxyacetonephosphate-acyltransferase, DHAP-AT, and phosphatidylethanolamine N-methyltransferase, PEMT) or in the intracellular trafficking of fatty acids (epidermal-fatty acid binding protein, E-FABP), were also quantified in the SH-SY5Y model. 


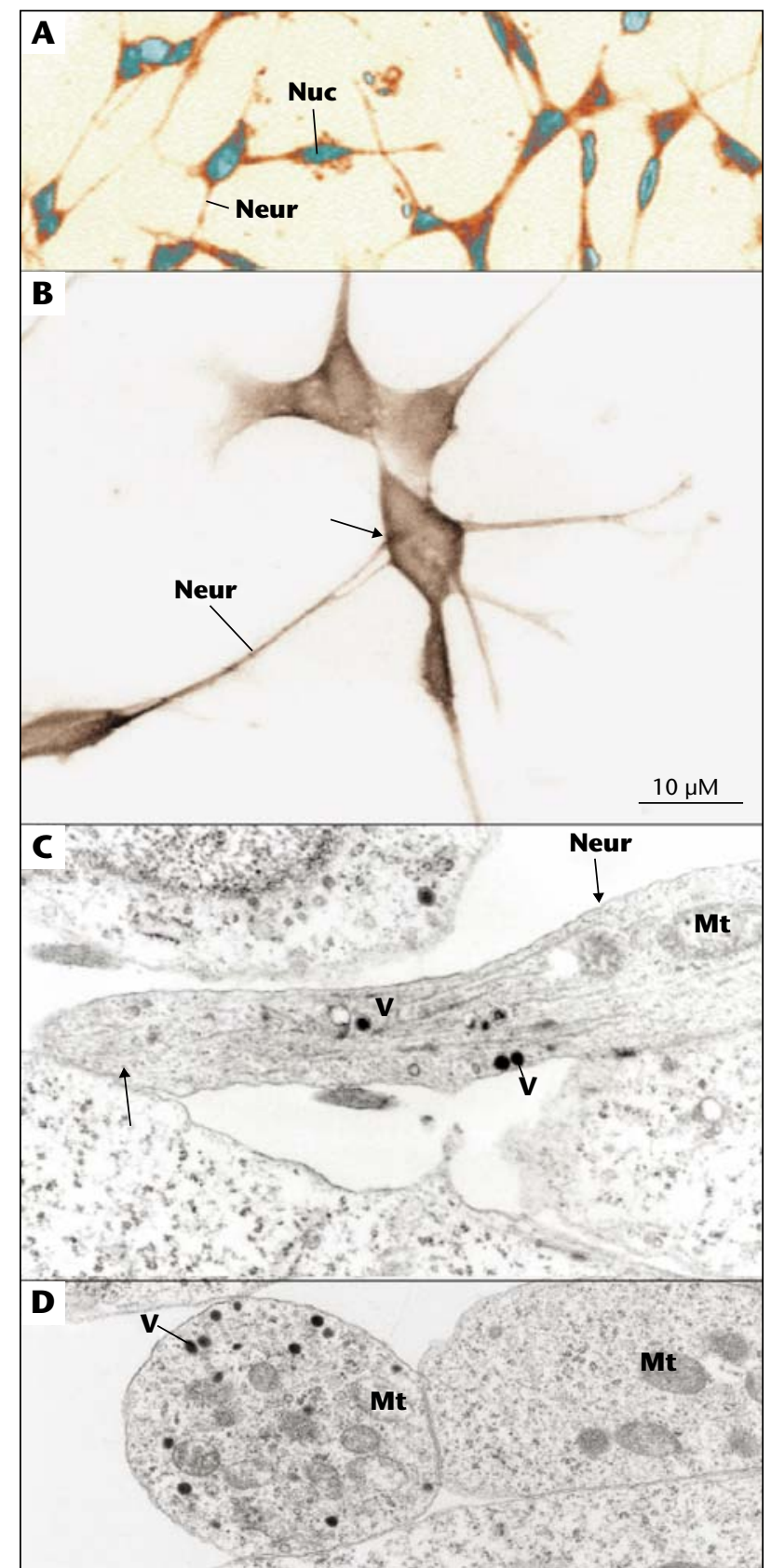

Figure 3. A) double-immunolabeling showing the presence of synaptotagmin I (a synaptic vesicle protein used as neuronal marker) and PPARS (a nuclear receptor) in SH-SY5Y cells. Synaptotagmin revealed by immunoperoxydase method appeared both in cytoplasm and neurites (Neur), PPARS nickel-enhanced diaminobenzidine staining is strictly localised in nucleus (Nuc). Differential colorization for peroxidase and nickel-labeling was performed using GraphicConverter ${ }^{T M}$ software. B) Synaptotagmin immunostaining of SH-SY5Y cells shows neurite extensions (Neur) and neurite-cell contact (arrow, scale bar $=10$ microns). C) electron microscopy (osmium staining) of SH-SY5Y cells showing a neurite extension (Neur) containing mitochondries (Mt) and neurotransmitter vesicles (V). Note the neurite-cell contact (arrow). D) transversal section of a neurite showing several catecholaminergic vesicles $(V)$ (original magnification $\times 22000$, photos: INRA).

\section{Material and methods}

\section{Cell cultures}

Neuroblastoma cells from the SH-SY5Y line were cultured in Falcon $75-\mathrm{cm}^{2}$ tissue culture flasks at $37^{\circ} \mathrm{C}$ in a $5 \%$ (by vol) $\mathrm{CO}_{2}$ atmosphere in DMEM supplemented with $10 \%$ heatinactivated fetal bovine serum [7]. In parallel, two types of brain endothelial cells were used: RBEC, which is a primary culture of endothelial cells isolated from the rat brain, and RBE4, an immortalized cell line issued from rat brain endothelial cells. RBEC were purified using the puromycin-based method described by Perriere et al. [8]. The microvessel fragments isolated from rat cerebral cortex were seeded on Petri dishes coated with type IV collagen ( 5 $\mu \mathrm{g} / \mathrm{cm}^{2}$ ) and incubated at $37^{\circ} \mathrm{C}$ in a saturated $5 \% \mathrm{CO}_{2}$ atmosphere in DMEM-F12 with $20 \%$ bovine plasma-derived serum. The RBEC were then purified using a specific treatment with puromycine $(4 \mu \mathrm{g} / \mathrm{mL})$ for 4 days. The RBE4 line is issued from immortalized RBEC [9]. RBE4 cells were grown at $37^{\circ} \mathrm{C}$ in a $5 \% \mathrm{CO}_{2}$ atmosphere in Falcon $75-\mathrm{cm}^{2}$ tissue culture flasks coated with rat-tail collagen in $\alpha$-MEM + glutamate enriched HAM's F10 supplemented with $10 \%$ heat-inactivated fetal calf serum.

The n-3 PUFAs, ALA, EPA, DPA or DHA, were directly added to the medium (containing $10 \%$ fetal calf serum) under the form of sodium salts. Graded concentrations (7 to $70 \mu \mathrm{M}$ ) of each fatty acid were used. The SH-SY5Y cells were cultured for $72 \mathrm{~h}$ in the supplemented medium. The RBEC and RBE4 cells were exposed to the fatty acid on the first day of culture and the supplemented medium was refreshed every 3 days throughout the culture. At the end of the cultures, the cells were trypsinised (SH-SY5Y cells) or washed twice with PBS-0.5\% BSA, scraped off (RBEC and RBE4) and collected by centrifugation.

\section{Animal study}

Wistar pregnant female rats deficient in $\mathrm{n}-3$ PUFA (first generation) were assigned to experimental groups supplemented with graded doses of dietary DHA (from 0 to $400 \mathrm{mg} / 100 \mathrm{~g}$ diet) [10]. These doses were obtained by adding different amounts of a microalgal oil containing about $43 \%$ of DHA (DHASCO; Martek Biosciences Corporation, Columbia, MD). At weaning of the pups ( $2^{\text {nd }}$ generation, 3 wk of age), the males were retained and fed for 5 wk the same diet as that of their dam's group. At 8 wk of age, the rats were sacrificed and their frontal cortex and hippocampus were dissected.

\section{Fatty acid analysis}

The total lipids were extracted from cells and from brain samples with $4 \mathrm{ml}$ chloroformmethanol containing $0.02 \mathrm{~g} / 100 \mathrm{~mL}$ butylhydroxytoluene as antioxidant [7]. The ethanolamine phosphoglycerolipids (EPG), the main phospholipid class rich in DHA, were isolated from total lipids by solid-phase extraction on a single-use 500-mg aminopropyl-bonded silica column [11]. The EPG fraction was dried under a nitrogen flux and its fatty acids were converted to methyl esters by reaction with metha- 
nol and boron trifluoride at $90^{\circ} \mathrm{C}$ for $20 \mathrm{~min}$ (transmethylation). The fatty acid methyl esters were separated and quantified by gas liquid chromatography [11]; the fatty acid composition was expressed as a weight percentage of total fatty acids in EPG.

\section{Quantification of mRNA expression in the SH-SY5Y cells}

The mRNAs encoding nuclear receptors or lipid metabolism genes were quantified by retrotranscription of total RNAs into CDNAs; the target-cDNAs were amplified by quantitative real time-PCR. Total RNAs were prepared using the Rneasy lipid tissue Midi kit (Quiagen). Aliquots of total RNA $(6 \mu \mathrm{g})$ were reversetranscribed at $25^{\circ} \mathrm{C}$ for $10 \mathrm{~min}$ and at $37^{\circ} \mathrm{C}$ for $2 \mathrm{~h}$ using the High Capacity cDNA Archive Kit (Applied Biosystems). Primer pairs for target genes were designed with the assistance of Primer Express 2.0 (Applied Biosystems). The SYBR Green fluorescence method was used to detect the amplicons, except for E-FABP, $\triangle 6-D$ and DHAP-AT whose CDNAs were amplified and detected using sets of specific primers and the TaqMan probe (SYBR Green master mix and TaqMan Assays-on-Demand, Applied Biosystems). Primer pairs were validated under the condition that their PCR efficiency was above 95\% (therefore, differences in abundance of different retrotranscripts are not due to differences in PCR efficiency). The housekeeping gene (GAPDH) was quantified using both SYBR Green and TaqMan to normalize the abundance of all retrotranscripts. The mean value $(n=3$ triplicates per flask, 3 flasks per treatment) of the threshold cycle number (Ct) for detecting the appearance of each amplicon was compared to that of GAPDH. The amount of mRNA of each target gene was expressed relative to the GAPDH abundance according to the equation:

[Target gene abundance/GAPDH abundance] $=1 / 10^{\mathrm{n}}$, with $\mathrm{n}=\Delta \mathrm{Ct} \cdot \log 2$ and $\Delta \mathrm{Ct}=\mathrm{Ct}$ target gene Ct GAPDH

\section{Results}

DHA incorporation in the membrane phospholipids of cells and rat brain areas - Development of a linear model for comparing the dose-dependent responses of living tissues and of cultured cells to graded doses of preformed DHA

Neuroblastoma cells cultured in standard conditions, i.e. with $10 \%$ fetal calf serum and without PUFA supplementation, have a low content of DHA (around 5\% in EPG) (figure 4). The

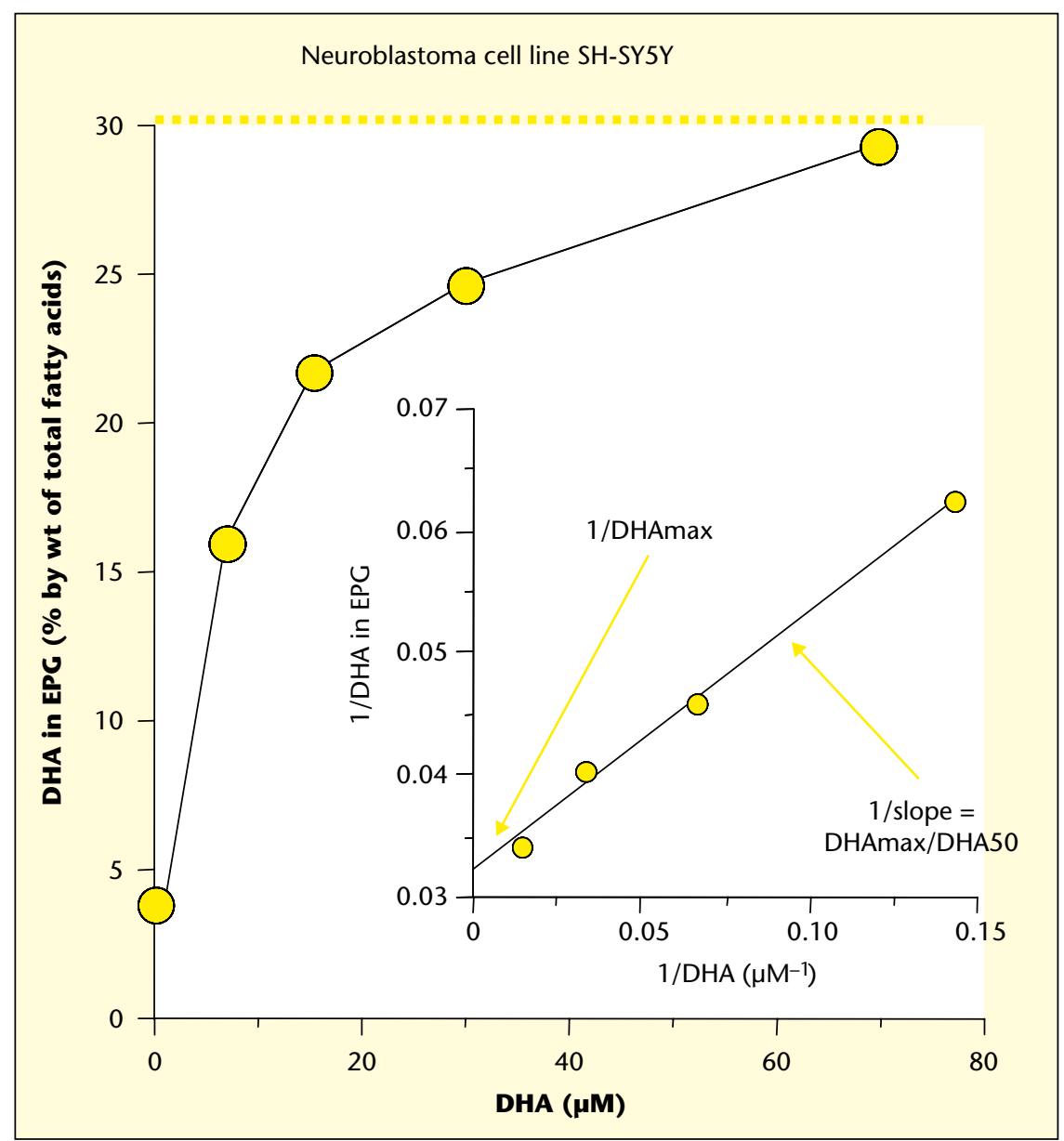

Figure 4. Docosahexaenoic acid (DHA, 22:6n-3) content (\% of total fatty acids) in ethanolamine phosphoglycerides (EPG) from the human neuroblastoma cell line SH-5YSY in response to increasing DHA doses in the culture medium (mean of 3 determinations). Double-reciprocal data are shown in the inset; theoretical maximal DHA concentration in membranes (DHAmax) is calculated by the determination of the ordinate at the origin and the avidity for DHA by that of the slope (DHAmax/DHA50).

dose-response curve for incorporation of preformed DHA was hyperbolic: the DHA content in EPG gradually increased from the basal value of $5 \%$ of total fatty acids to the plateau-value of $30 \%$ in cells grown in a medium supplemented with $70 \mu \mathrm{M} \mathrm{DHA}$ (figure 4). The dose-response curve for DHA incorporation in the EPG of rat frontal cortex and hippocampus produced a similar pattern of incorporation with $26-28 \%$ of DHA at the upper dietary dose (400 mg $\mathrm{DHA} / 100 \mathrm{~g}$ diet) (data not shown). We used a linear model to determine and compare the theoretical plateau-values (DHAmax) in the in vitro and in vivo models [10]. It consists in plotting the reciprocal of the DHA content $(1 / D H A)$ in EPG against the reciprocal of the dose $(1 /$ dose $)$ expressed in $(\mu \mathrm{M} \mathrm{DHA})^{-1}$ or in (mg DHA/100 $\mathrm{g} \mathrm{diet}^{-1}$ (figure 4). The theoretical DHAmax content in EPG is drawn from the reciprocal value of the ordinate at the origin (equal to 1/DHAmax) of the straight line. Moreover, the reciprocal value of the slope (1/slope) defines the ratio of DHAmax to
DHA50, the DHA50 being the dose of preformed DHA required to match half the value of DHAmax in EPG. Thus, comparison between cell models and living tissues is possible through the comparison of the DHAmax and of the DHAmax/DHA50 ratio, reflecting the 'avidity' of cells and tissues for preformed DHA [10]. The data showed that the avidity of SH-SY5Y cells for preformed DHA was algebraically comparable to that of the rat frontal cortex and hippocampus, with the condition that concentrations of DHA in the medium and in the diet were expressed in $\mu \mathrm{M}$ and in $\mu \mathrm{mol} \mathrm{DHA} / 10 \mathrm{~g}$ diet, respectively (table 1). Thus, we showed that the SH-SY5Y cells are able to use preformed DHA, as do living tissues, for their membrane biogenesis, indicating that the uptake of DHA from the medium, the acyl-CoA synthesis and the acyltransferase activities are fully active in these cells. The same model of preformed DHA incorporation was applied to the rat brain endothelial cells. The doseresponse curve of RBEC was different from that 
Table 1. Comparison of the theoretical maximal DHA concentration (DHAmax) and the avidity for DHA in ethanolamine phosphoglycerides (EPG) from cell models and rat tissues. Data are the mean of 3 (cell models) or 4 (rats) individual determinations. DHA50 values in the cells and the rat tissues are expressed in $\mu \mathrm{mol} / \mathrm{L}$ and $\mu \mathrm{mol} / 10 \mathrm{~g}$ diet, respectively.

\begin{tabular}{|lcc|}
\hline & $\begin{array}{c}\text { DHAmax } \\
\text { (\% of total fatty acids) }\end{array}$ & $\begin{array}{c}\text { Avidity } \\
\text { (DHAmax/DHA50) }\end{array}$ \\
\hline Neuroblastoma human cell line SH-SY5Y & 32.7 & 5.2 \\
Rat frontal cortex & 28.8 & 5.7 \\
Rat hippocampus & 26.2 & 5.1 \\
Primary rat brain endothelial cells (RBEC) & 30.3 & 2.8 \\
Rat brain endothelial cell line (RBE4) & 33.7 & 5.0 \\
\hline
\end{tabular}

of neuroblastoma cells, leading to lower DHAmax and avidity, while the RBE4 response superimposed that of SH-SY5Y cells (data not shown). The corresponding parameters for DHA incorporation in RBEC and RBE4 are reported in table 1.

\section{Membrane incorporation of neoformed $n$-3 long-chain PUFAs in SH-SY5Y cells}

The SH-SY5Y cells were incubated with graded doses of each of the metabolic precursors of DHA (ALA, EPA or DPA) and the incorporation of the neoformed $n-3$ PUFA in EPG was analysed. The resulting dose-response curves are shown in figure 5. It appeared that increasing the doses of ALA in the medium gradually increased the membrane incorporation of neoformed EPA and DPA. This pattern clearly indicates that the endoplasmic reticulum pathway of synthesis (up to DPA) is active in these cells. Regarding neoformed DHA, the response of cells to low concentrations of ALA $(<15 \mu \mathrm{M})$ showed an initial increase of incorporation which peaked at $10 \%$ DHA in EPG (i.e. a gain of $30 \%$ DHA compared to control value) followed by a decreased incorporation at concentrations of ALA greater than $15 \mu \mathrm{M}$ (figure 5). The same

pattern was observed with EPA supplementation (data not shown), consisting in a gradual increase of EPA and of its elongation product DPA in EPG, whereas the neoformed DHA followed a bell-shaped curve with a peak of incorporation ( $9 \%$ of total fatty acids) at $15 \mu \mathrm{M} \mathrm{EPA}$ [7]. When the cells were cultured with graded doses of DPA, both the neoformed EPA (produced through the retroconversion pathway) and DPA gradually increased in EPG. The bellshaped curve of neoformed DHA peaked at 30 $\mu \mathrm{M}$ DPA with a maximum of DHA incorporation equal to $12 \%$ of total fatty acids (figure 5).

\section{mRNA expression of lipid metabolism genes in SH-SY5Y cells}

The basal relative abundances of the mRNAs encoding proteins and enzymes of the endoplasmic reticulum- and peroxisomal-pathways, and those of the mRNAs encoding the cognate nuclear receptors, are reported in figure 6 . The SH-SY5Y cells constitutively express the mRNA of these lipid metabolism genes, whose relative abundances (normalized to that of GAPDH) range from $10 \%$ (PEMT, ELOVL4, DBP) to $85 \%(\Delta 6 \mathrm{D})$. The basal levels of the DBP and LBP transcripts were among the lowest that we

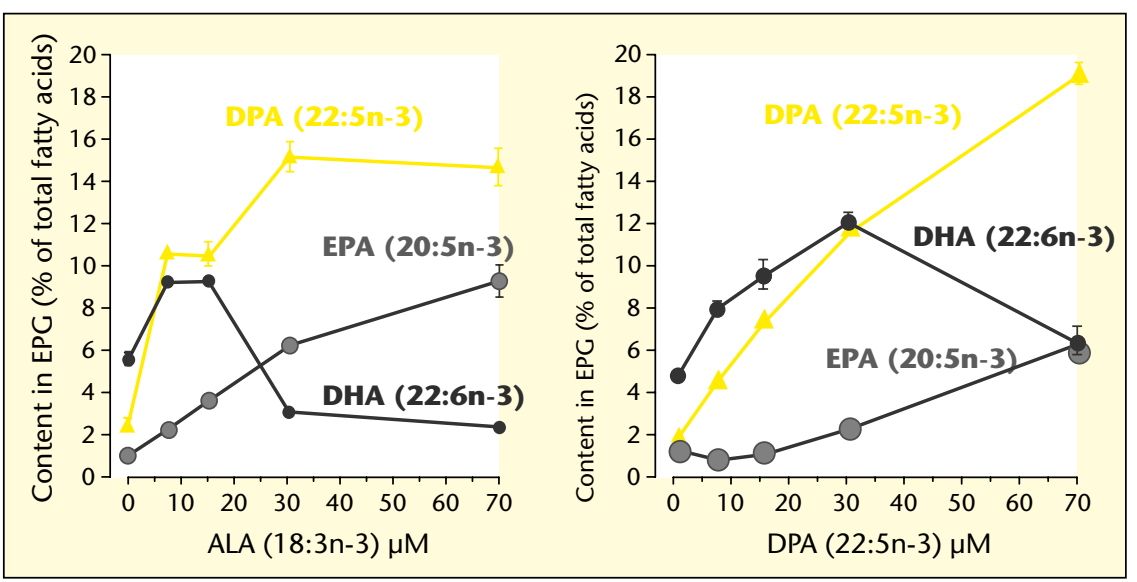

Figure 5. Incorporation of n-3 long chain n-3 PUFAs in ethanolamine phosphoglycerides (EPG) from the human neuroblastoma cell line SH-5YSY cultured with graded concentrations of ALA (18:3n-3) or DPA (22:5n-3) (mean \pm SD of three separate cultures). determined, suggesting that the transcription of peroxisomal enzymes may be constitutively limiting in these cells. The SH-SY5Y cells also express transcription factors known to be involved in the regulation of lipid metabolism, i.e. the PPARs, mainly the $\alpha-$ and $\beta / \delta$-isotypes, and RXR $\alpha$.

\section{Discussion}

We have examined the capacity of a neural cell model, the human neuroblastoma cell line SH-SY5Y, to produce the long-chain n-3 PUFAs issued from the metabolic conversion of ALA, the essential precursor. Our data show that the SH-SY5Y model is particularly appropriate for studying the regulation of DHA synthesis. These cells avidly incorporate exogenous (preformed) DHA following a dose-dependent response that is very similar (in terms of substrate avidity and plateau-value) to that of rat brain areas. On the other hand, the DHA synthesis from its upstream precursors is efficient but limited in the SH-SY5Y cells. We suggest that the production of neoformed DHA is 'bottlenecked' in these cells, resulting in the membrane accumulation of neoformed EPA and DPA at high concentration of supplemental ALA. These data on this cell model may have a physiological significance, since a similar effect (accumulation of EPA and DPA) is observed in the blood lipids of humans receiving high doses of ALA (figure 2) [4].

It must be emphasized that cells cultured in standard conditions have very low DHA content in their phospholipids, which is equivalent to the physiological state of a chronical deficiency in dietary n-3 PUFAs $[10,12,13]$. Low DHA contents have been reported from miscellaneous neural cell models [7, 11, 14-16]. Thus, it appears that the different serum-based culture media do not provide enough DHA, and that supplementation of the medium with preformed DHA is needed to sustain physiological levels of DHA into the membrane phospholipids of cultured cells. Our data showed that the incorporation of exogenous DHA into the membrane phospholipids (EPG) of SH-SY5Y cells supplied with graded amounts of preformed DHA followed a dose-response hyperbolic curve from a minimum content to a plateau value, thereby reproducing the restauration of the DHA status in the rat brain [17]. The pattern of DHA incorporation was featured (in vivo and in vitro) by calculating two parameters, DHAmax and DHA50. The DHAmax values (expressed in \% by weight of total fatty acids in EPG) of neuroblastoma cells (32.7), RBEC (30.3) and RBE4 cells (33.7) were similar to those of the frontal cortex (28.8) and of the hippocampus (26.2). The ratios of DHAmax to DHA50 were identical in neuro- 


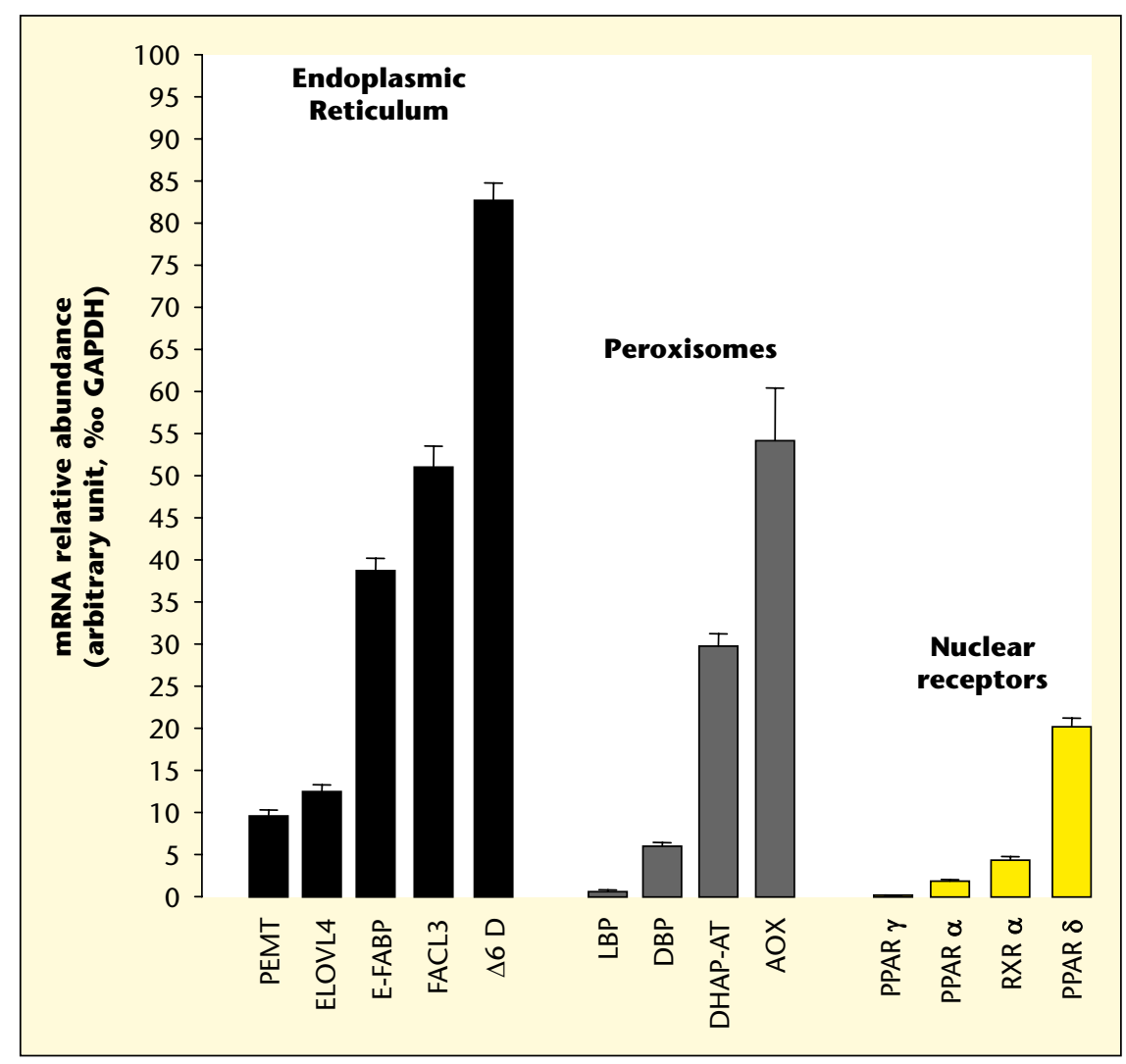

Figure 6. Relative abundance of mRNA encoding lipid metabolism and cognate nuclear receptor genes in the human neuroblastoma cell line SH-SY5Y. Cells were cultured in standard condition with 10\% fetal bovine serum. Each value is the mean $\pm S D$ of three culture flasks. Endoplasmic reticulum genes: phosphatidylethanolamine $\mathrm{N}$-methyltransferase, PEMT; elongase of very long chain fatty acids, ELOVL4; human epidermal fatty acid binding protein, E-FABP; fatty acid-COA ligase, FACL3; $\triangle 6$ desaturase, $\triangle 6$ D. Peroxisomal genes: L- and D-bifunctional proteins, LBP and DBP; dihydroxyacetone-phosphate acyltransferase, DHAP-AT; acyl-CoA oxidase, AOX. Cognate nuclear receptor genes: peroxisome proliferator-activated receptors (isoforms PPAR- $\alpha,-\beta$ and $-\delta$ ); retinoid $x$ receptor $\alpha(R X R \alpha)$. GAPDH, glyceraldehyde-3-phosphate dehydrogenase.

blastoma (5.2) and RBE4 (5.0) cells indicating that these two lines have the same avidity for preformed DHA. The DHAmax/DHA50 ratio value of 2.8 in RBEC indicated that the avidity of these primary culture cells for DHA is around 1.8-time lower than that of the two cell lines (RBE4 and SH-SY5Y). Differential avidity of RBEC and RBE4 for DHA may reflect the physiological specificity of RBEC to preferentially use arachidonic acid (20:4n-6), not DHA, for membrane biogenesis.

The expression of the dietary dose in $\mu \mathrm{mol}$ $\mathrm{DHA} / 10 \mathrm{~g}$ diet led to a DHAmax/DHA50 ratio value of 5.7 and 5.1 in the rat cortex and hippocampus, respectively, that may be compared with the value of 5.0-5.2 in cultured cells (table 1). This algebraic identity suggests that a physiological equivalence ( $\mu \mathrm{mol} \mathrm{DHA}$ /liter vs $\mu \mathrm{mol} \mathrm{DHA} / 10 \mathrm{~g}$ diet) can be established between the DHA concentration in the culture medium and in the diet. From this model, it was also inferred that a dietary supply of 10 -fold the DHA50 of brain EPG should be centration. Thus, these cells have the capacity to complete the synthesis of DHA at low concentration of precursors, through the endoplasmic reticulum and peroxisomal pathways. This finding is notable since it is generally thought that the synthesis of DHA from n-3 PUFAs is limited in cultured brain cells at the elongation of DPA [16]. However, increasing the concentrations of supplemental n-3 precursors above $15 \mu \mathrm{M}$ (ALA and EPA) or above $30 \mu \mathrm{M}$ (DPA) resulted in a sharp decrease of the newly formed DHA while EPA and DPA continued to increase. The membrane accumulation of EPA and DPA at high concentration of precursor is compatible with the hypothesis that the downstream pathway of DHA synthesis is 'bottlenecked'. Therefore, we assume that production of membrane DHA by SH-SY5Y cells is limited at the stage of DHA terminal synthesis, probably at the peroxisomal step, resulting in the overflow in membranes of its parent fatty acids, EPA and DPA. Since the mRNA abundances of the peroxisomal LBP and DBP are particularly low in standard conditions of culture, we suggest that the low expression of proteins or enzymes specifically related to the peroxisomal function could form a 'metabolic bottleneck'. We conclude that the SH-SY5Y cells provide an appropriate in vitro model for studying the regulation of the PUFA metabolism, especially at the level of the gene transcription of peroxisomal enzymes. The stimulation of the peroxisomal pathway could be the key for completing the synthesis of DHA from its dietary precursors.

Acknowledgements. The authors thank Françoise Roux and Nicolas Perrière (INSERM U705-CNRS UMR 7157, Hôpital Fernand Widal, Paris) for the gift of RBE4 cells and help for RBEC purification. $90 \%$ of the DHAmax in the brain [10].

The capacity of SH-SY5Y cells to produce longchain metabolites was evidenced by the incorporation of EPA, DPA and DHA into the EPC fraction. Their membrane incorporation is the final outcome of the global process of n-3 PUFA metabolism, including long-chain conversion, acylation, phospholipid metabolism, and turnover $[7,11]$. Accumulation of DPA clearly indicated that the metabolic process is operating in SH-SY5Y cells, and their mRNA profile supports this conclusion. We emphasize that the $\Delta 6$-desaturase, which catalyses the first step of the long-chain PUFA synthesis [18], was expressed to a relatively high mRNA level. Both the accumulation of neoformed DPA and the mRNA relative abundance of $\Delta 6$-desaturase suggest that $\Delta 6$-desaturation is not rate-limiting in these cells. The SH-SY5Y cells cultured with ALA, EPA or DPA also produced DHA, although the production of neoformed DHA was restricted to a critical window of precursor con-

\section{REFERENCES}

1. ALESSANDRI JM, GUESNET $P$, VANCASSEL $S$, et al. Polyunsaturated fatty acids in the central nervous system: evolution of concepts and nutritional implications throughout life. Reprod Nutr Dev 2004; 44: 509-38.

2. CHALON S. Omega-3 fatty acids and monoamine neurotransmission. Prostaglandins Leukot Essent Fatty Acids 2006; 75: 259-69.

3. GUESNET P, ALESSANDRI JM. Acides gras polyinsaturés du lait et développement du système nerveux central du nouveau-né. Cah Nutr Diét 1995; 30: 109-11.

4. ARTERBURN LM, HALL EB, OKEN H. Distribution, interconversion, and dose response of $n-3$ fatty acids in humans. Am / Clin Nutr 2006; 83: 1467S-1476S. 
5. BURDGE GC. Metabolism of alpha-linolenic acid in humans. Prostaglandins Leukot Essent Fatty Acids 2006; 75: 161-8.

6. JENSEN CL, CHEN H, FRALEY JK, ANDERSON RE, HEIRD WC. Biochemical effects of dietary linoleic/alpha-linolenic acid ratio in term infants. Lipids 1996; 31: 107-13.

7. LANGELIER B, ALESSANDRI JM, PERRUCHOT MH, GUESNET P, LAVIALLE M. Changes of the transcriptional and fatty acid profiles in response to $\mathrm{n}-3$ fatty acids in SH-SY5Y neuroblastoma cells. Lipids 2005; 40: 719-28.

8. PERRIERE N, DEMEUSE $P$, GARCIA E, et al. Puromycin-based purification of rat brain capillary endothelial cell cultures. Effect on the expression of blood-brain barrier-specific properties. J Neurochem 2005; 93: 279-89.

9. ROUX F, DURIEU-TRAUTMANN O, CHAVEROT $\mathrm{N}$, et al. Regulation of gamma-glutamyl transpeptidase and alkaline phosphatase activities in immortalized rat brain microvessel endothelial cells. / Cell Physiol 1994; 159: 10113.
10. ALESSANDRI JM, POUMES-BALLIHAUT C, LANGELIER B, et al. Incorporation of docosahexaenoic acid into nerve membrane phospholipids: bridging the gap between animals and cultured cells. Am J Clin Nutr 2003; 78: 702-10.

11. LANGELIER B, FURET JP, PERRUCHOT MH, ALESSANDRI JM. Docosahexaenoic acid membrane content and mRNA expression of acyl$\mathrm{CoA}$ oxidase and of peroxisome proliferatoractivated receptor-delta are modulated in $Y 79$ retinoblastoma cells differently by low and high doses of alpha-linolenic acid. / Neurosci Res 2003; 74: 134-41.

12. XIMÈNES DA SILVA A, LAVIALLE F, GENDROT G, GUESNET PH, ALESSANDRI JM, LAVIALLE M. Glucose transport and utilization are altered in the brain of rats deficient in $n-3$ polyunsaturated fatty acids. / Neurochem 2002; 81: 132837.

13. AÏD $S$, VANCASSEL $S$, POUMÈS-BALLIHAUT C, CHALON S, GUESNET PH, LAVIALLE M. (n-3) polyunsaturated fatty acid deficiency led to cholinergic changes in rat hippocampus. / Lipid Res 2003; 44: 1545-51.
14. AKBAR M, CALDERON F, WEN Z, KIM HY. Docosahexaenoic acid: a positive modulator of Akt signaling in neuronal survival. Proc Natl Acad Sci USA 2005; 102: 10858-63.

15. CHAMPEIL-POTOKAR G, DENIS I, GOUSTARDLANGELIER B, ALESSANDRI JM, GUESNET PH, LAVIALLE M. Astrocytes in culture need docosahexaenoic acid (DHA) to restore the $n-3 / n-6$ polyunsaturated fatty acid (PUFA) balance in their membrane phospholipids. Neurosci Res 2004; 75: 96-106.

16. INNIS SM, DYER RA. Brain astrocyte synthesis of docosahexaenoic acid from n-3 fatty acids is limited at the elongation of docosapentaenoic acid. J Lipid Res 2002; 43: 1529-36.

17. GUESNET $P$, ALASNIER C, ALESSANDRI JM, DURAND G. Modifying the n-3 fatty acid content of the maternal diet to determine the requirements of the fetal and suckling rat. Lipids 1997; 32: 527-34.

18. GUILLOU H, D'ANDREA S, RIOUX V, JAN S, LEGRAND $P$. The surprising diversity of Delta6desaturase substrates. Biochem Soc Trans 2004; 32: 86-7. 